\title{
Simulations and experiments on vibration damping for zoom-holography and nano-scanning at the GINIX
}

\author{
Markus Osterhoff ${ }^{1}$, Peter Luley ${ }^{1}$, Michael Sprung ${ }^{2}$, Tim Salditt ${ }^{1}$ \\ ${ }^{1}$ Institut für Röntgenphysik, University of Göttingen, Friedrich-Hund-Platz 1, D-37077 Göttingen \\ ${ }^{2}$ DESY Photon Science, Notkestraße 85, D-22607 Hamburg
}

\begin{abstract}
The Göttingen Instrument for Nano-Imaging with X-ray (GINIX) is a holography endstation located at the P10 coherence beamline at PETRA III, designed and operated by the University of Göttingen in close collaboration with DESY Photon science Hamburg [1-2]. GINIX is designed as a waveguide based holography experiment with a Kirkpatrick-Baez nanofocus. Its versatility has stimulated a great manifold of imaging modalities. Today, users choose the GINIX setup not only for its few nm coherent waveguide beams (e.g. for ptychography or holography), but also to carry out scanning SAXS measurements to probe local anisotropies with sub-micron real-space and even higher reciprocal space resolution. In addition, it is possible to combine different detectors for e.g. simultaneous SAXS/WAXS and fluorescence measurements [3].

We summarise our ongoing efforts to reduce vibrations in the setup, and present latest experimental results obtained with GINIX, focusing on the unique capabilities offered by its versatile and flexible design. The overview includes results from different imaging schemes such as waveguide based zoom-tomography and user examples in WAXS geometry. We show how to correlate complementary techniques like holography and scanning SAXS and present first results obtained using a new fast sample scanner for Multilayer Zone Plate imaging.
\end{abstract}

Keywords: X-ray imaging, endstations, zoom-holography, nano-scanning

\section{INTRODUCTION}

New fabrication techniques and methods have pushed the quality of X-ray optics. While a few years ago a "nano-focus" endstation achieved focal spot sizes on the order of hundreds of nano metre, nowadays the beam sizes well below $100 \mathrm{~nm}$ are used in a routine base, and the single nano metre beam size is expected. With smaller and brighter spot sizes, vibrations and drift of the end-station itself have be addressed - vibrational amplitudes of tens of nano metre are not an exception at most beamlines.

Here we address the vibrations of the GINIX setup located at the P10 coherence beamline at PETRA III, DESY Hamburg. Vibrations are measured interferometrically, and a mechanical model of the set-up is analysed using simulation software based on these measured data. We describe several measures that have been taken to improve the situation and damp the vibrations. Then, we summarise the recent experiments performed at GINIX, with a special emphasis on scanning nano-SAXS and waveguide based holo-tomography. 


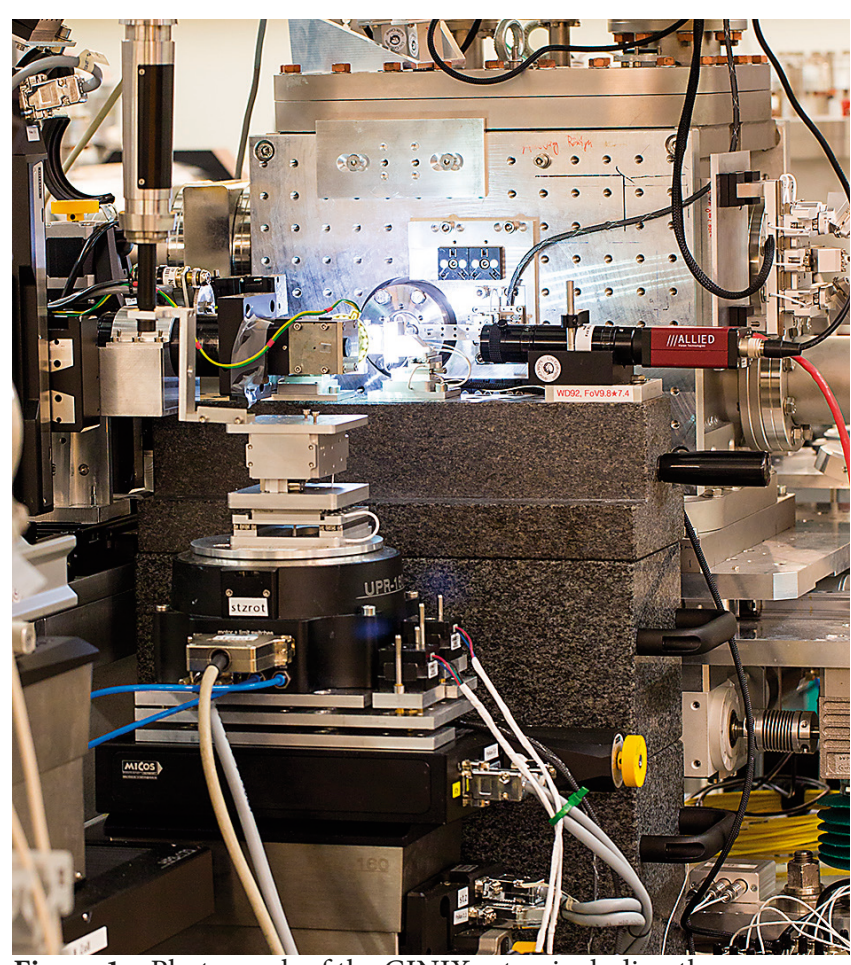

Figure 1a. Photograph of the GINIX setup including the new high-resolution granite for nano-scale hard $\mathrm{x}$-ray tomography with waveguide based holography.

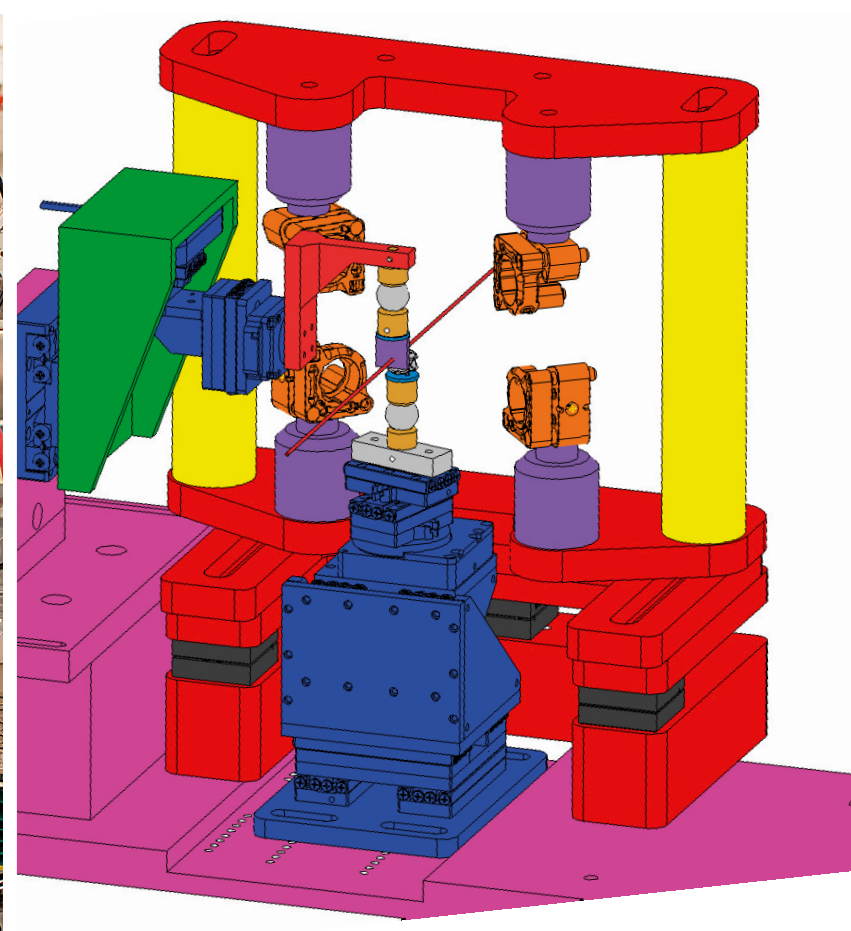

Figure 1b. CAD model of new high-resolution waveguide based holo-tomo tower, now including interferometric control both for waveguide and for the sample position.

\section{UPGRADES: REDUCING VIBRATIONS}

\section{Initial Design}

The GINIX setup has been designed and commissioned for waveguide based holography, at a resolution of $100 \mathrm{~nm}$ and better. This is reflected in the design of the Kirkpatrick-Baez mirrors (focal spot size around $300 \mathrm{~nm}$ [2]) and the guiding core sizes of typical X-ray waveguides that can below $100 \mathrm{~nm}$. Also the pixel size of detectors commonly used about ten years ago align with this resolution goal. In the meantime, however, waveguide fabrication has improved a lot at our group; nowadays, $2 \mathrm{D}$ guiding cores of sub- $40 \mathrm{~nm}$ can be achieved, and using tapered layouts the available coherent flux for nano-imaging could be increased to $10^{10}$ photons per second and more [4]; a higher flux is possible using $1 \mathrm{D}$ waveguides. Recently, the GINIX has undergone several upgrades to improve on vibrations, see e.g. the new high-resolution tomographic tower depicted in Fig. 1.

\section{Measurements}

Designed for relaxed resolution goals, the original GINIX has hit quite a barrier in imaging at sub-50 nm demagnified pixel size. This is due to vibrations of the setup; interferometric measurements show significant peaks $(12 \mathrm{~nm} @ 78 \mathrm{~Hz})$ at the granite base and $(40 \mathrm{~nm} @ 12 \mathrm{~Hz})$ at the optical breadboard (below sample stages). Typical measurements before and after the improvements mentioned below are shown in Fig. 2a and Fig. 2b. These and similar measurements have been taken with three different interferometer systems: SIOS Vibrometer (SIOS Meßtechnik GmbH, Ilmenau, Germany), FPS 3010 (Attocube GmbH, München, Germany), Picoscale (SmarAct GmbH, Oldenburg, Germany). They all give similar results, but differ e.g. in data rate, size of the focusing head, and ease of setup / calibration.

Interferometric positions are only relative between the (possibly vibrating) focusing head and the point of interest. To confirm the accuracy of the different instruments, also the mountings and pillars have been analysed. In addition, we have acquired an acceleration sensor Accelerometer 731-207 (by Wilcoxon Research Inc., Meggitt PLC, Dorset, UK; including Dataq DI-155 USB data logger), which is capable to measure nm-small amplitudes over a wide frequency range. In Fig. 2c, selected measured vibrations are included into a (very simple) CAD model of the GINIX setup, highlighting mainly the lateral ( $y$-direction) movements. 

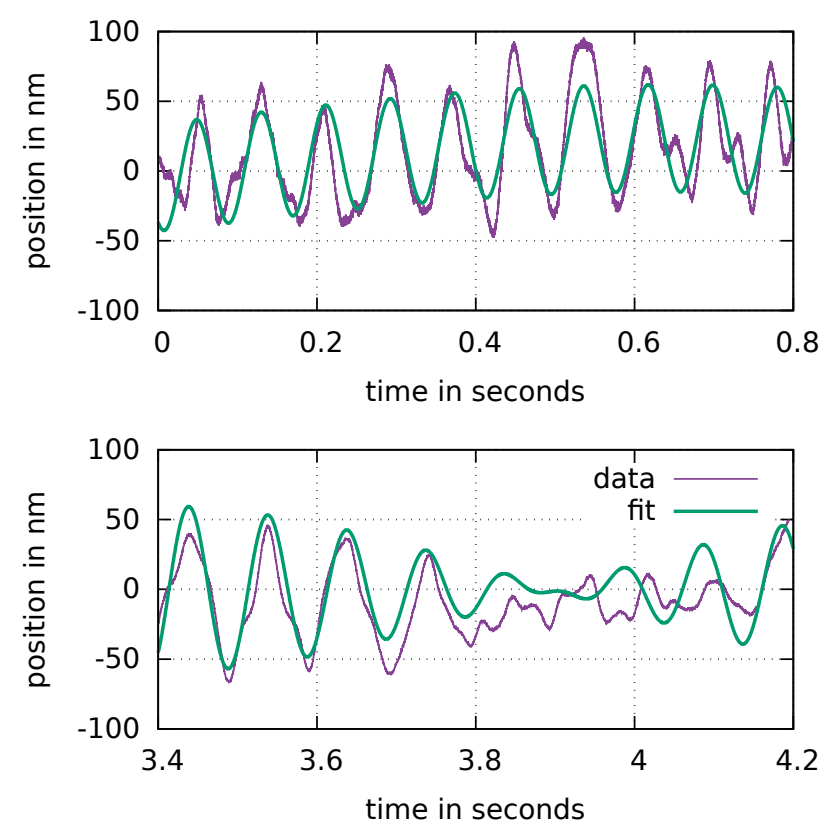

Figure 2a,b. Interferometry of optical breadboard, before (top) and after (bottom) the improvements described in the text. Typical amplitudes could be reduced by about $20 \%$.

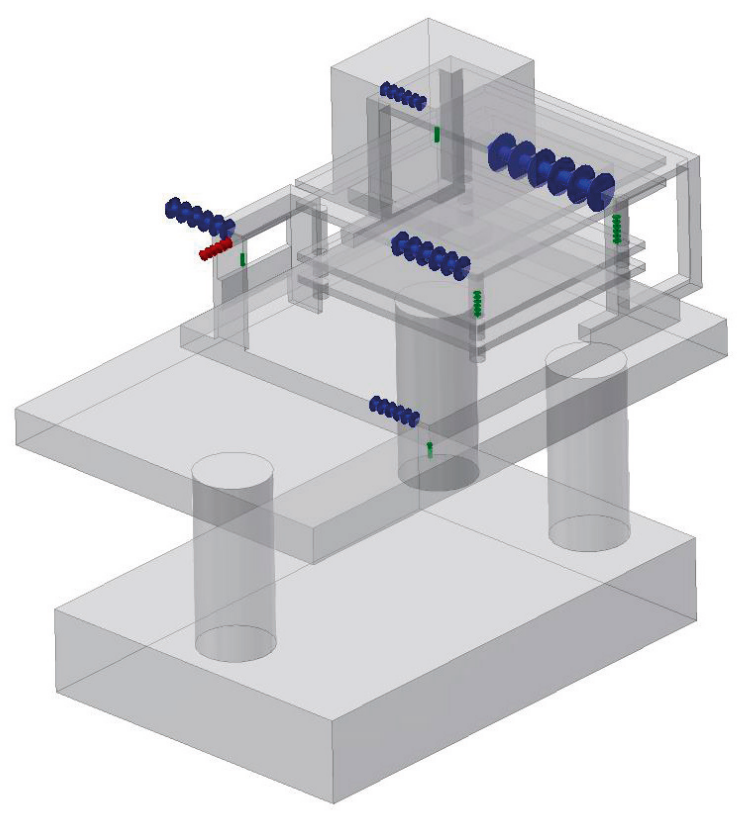

Figure 2c. Simple CAD view of the GINIX, quantifying vibrations: cross sections of arrows show the amplitude, length shows the frequency.

\section{Simulations}

These acceleration and vibration measurements are used as "ground truth" for simulations of and computer-aided optimisations of the mechanical components using SimulationX by ITI.

For simulation, the GINIX is abstracted to these minimal mechanical components: linear drives, bearings and inertial masses. Drives are simulated as spring-damper combinations. A layout of the design is shown in Fig. 4. The parameters of the components are measured as accurately as possible, and the tolerance is used to adjust the simulation results to measured vibrations and amplitudes, compare Fig. 4 to Fig. 2a.

One problem of the simulations is the modelling of measured noise, which overlays the eigenfrequencies. The noise model has already been optimised a lot; for the future, this will be further improved with more experimental data.

\section{Improvements}

To improve imaging resolution, several steps have been carried out. First, the influence of additional mass $(198 \mathrm{~kg}$ of $\mathrm{Pb}$ blocks) on the granite base and on the optical breadboard can reduce the $78 \mathrm{~Hz}$ vibrations, mainly by detuning resonance frequencies. The more prominent $12 \mathrm{~Hz}$ peak at the sample stage is, however, not affected.

We have taken the following measures that did improve in fact improve the level of vibrations:

- the housing has been separated from the optical setup,

- all controllers are moved from the granite base to the top of the housing,

- flexible re-mounting of all cables;

- installation of mass damper as vibration absorber.

Using the first three measures, vibrations could be reduced by approximately $20 \%$. During commissioning and tuning of the mass damper, strong fluctuations of the vibrations have been found. It has turned out that the main influence on vibration amplitudes comes from the general working hours on the DESY campus: Fig. 3 shows the main amplitudes for different 


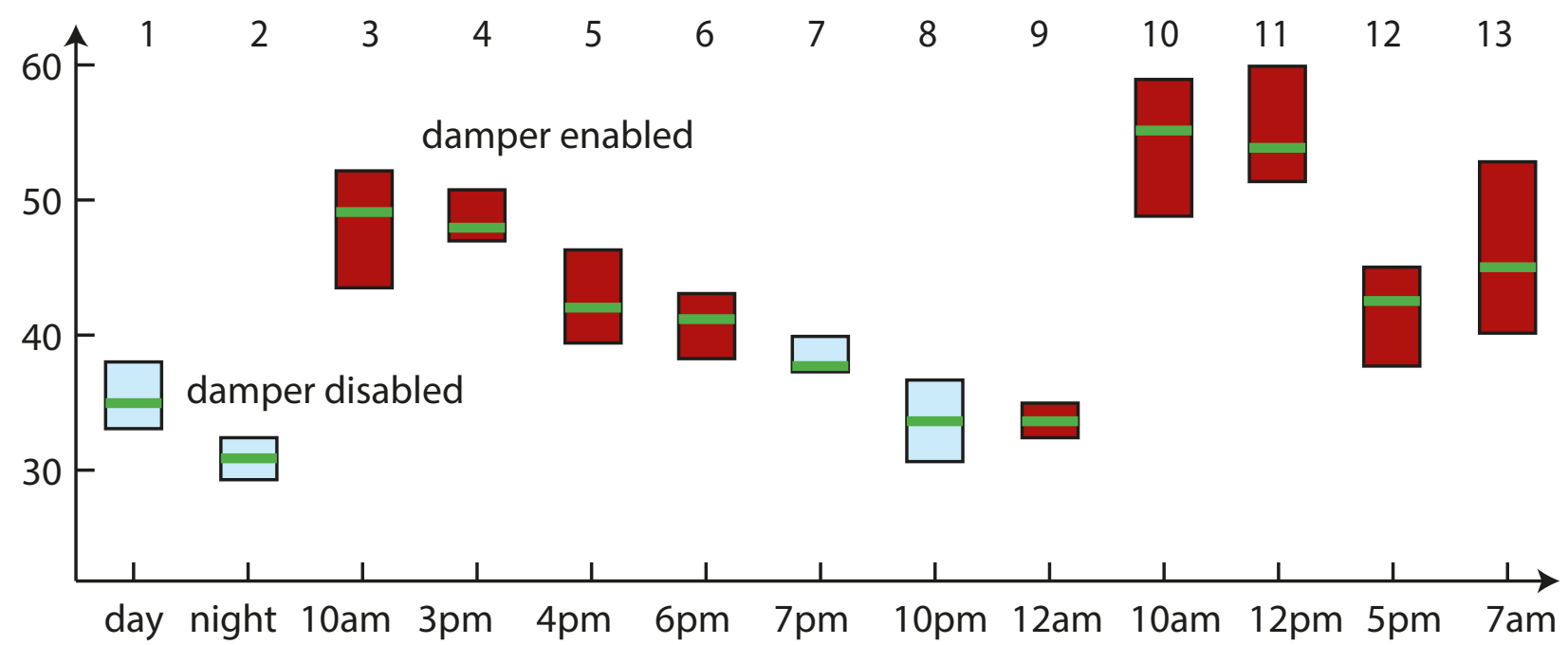

Figure 3. Vibration amplitudes during a day-cycle, with mass damper enabled (dark red) and disabled (light blue). Note the small influence of the damper between e.g. point 8 vs. 9, compared to the large jump from midnight to morning, point 9 vs. 10. Although simulations promise that the mass damper should reduce the most prominent vibrations by about $40 \%$, the shift from night to day introduces far larger amplitudes.
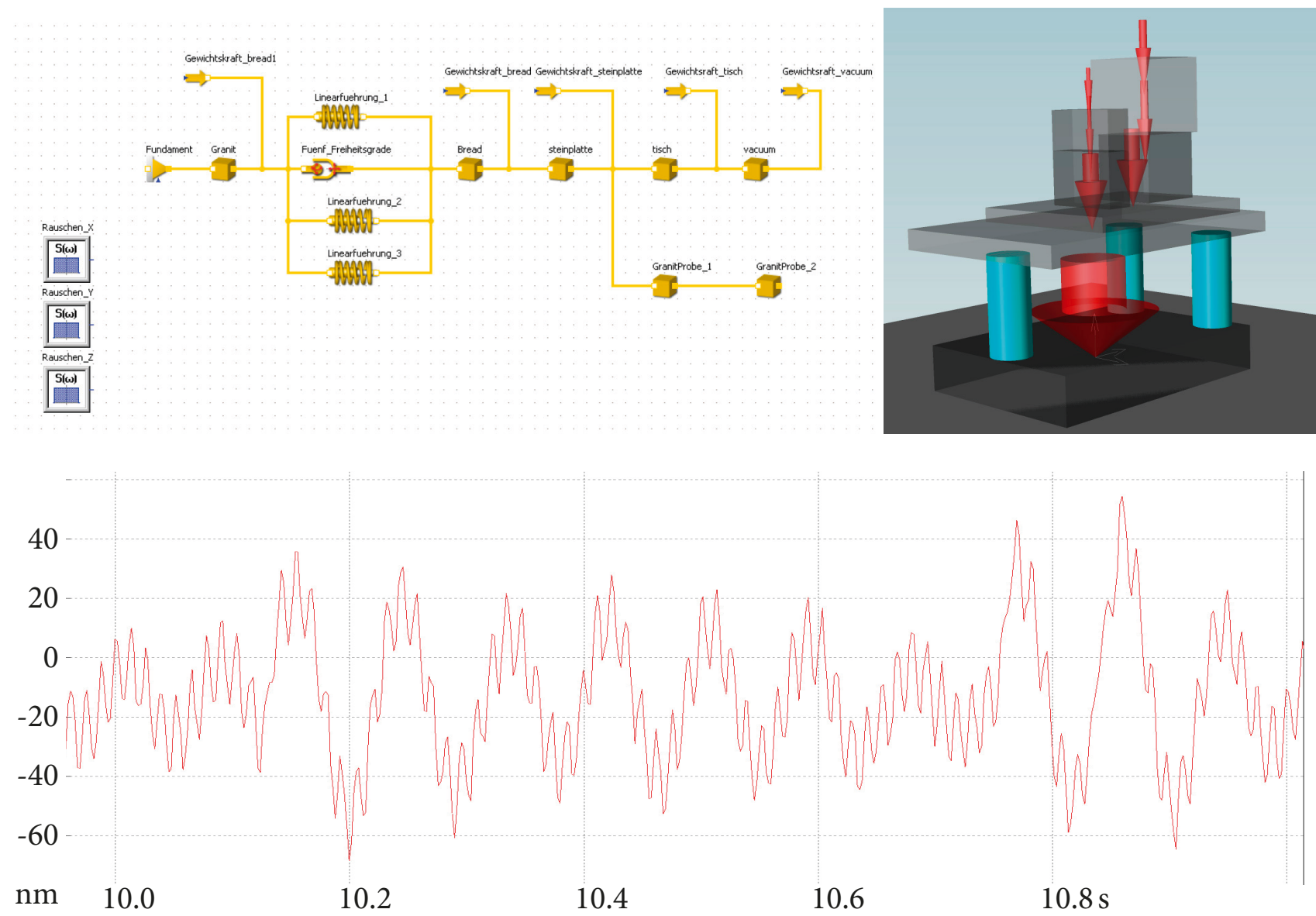

Figure 4. Numerical study of the eigenfrequencies of a minimal GINIX model: The simulation reproduces the known eigenmodes at $12 \mathrm{~Hz}$ and $78 \mathrm{~Hz}$ quite well; in future, the model of white noise will be improved. Based on these studies, the damping of vibrations is first optimised numerically, and then tested at the beamline. 
settings of the damper (dark red: with damper; light blue: without damper), but also at different times during two days. As can be seen, the damper has virtually no effect (compare e.g. points 6 vs. 7 and 8 vs. 9), while vibrations change significantly from night do day (see e.g. points 9 vs. 10).

\section{Less effective attempts}

In addition to the mechanical damper, also additional air springs, rubber mounts, and foams have been tried - in part at the beamline, in part at home. Also the active damping system Nano_20 (Accurion GmbH, Göttingen, Germany) has been integrated for a test run. As it turns out, none of these methods is able to further reduce vibrations significantly.

\section{Summary and future plans}

The GINIX setup is by design very flexible, offering different measurement procedures to users. But this flexibility comes at the cost of vibrations with amplitudes between $25 \mathrm{~nm}$ and $50 \mathrm{~nm}$, mainly at $12 \mathrm{~Hz}$. We could reduce the amplitude by about $20 \%$ by rather easy means; but the largest variance is so far given by general working hours on site. In future, the simulations will be improved by better parameters (e.g. additional spring constants, degrees of freedoms) and experimental data on vibrations; also the influence of rotations vs. translations need to be included.

\section{SCANNING SAXS}

\section{Overview}

Scanning SAXS experiments utilise a nano-focused X-ray beam for moderate to high spatial resolution, and a large photon counting detector for high reciprocal, or resolution in $q$-space. At the GINIX, Kirkpatrick-Baez mirrors focus down to about $300 \mathrm{~nm}$ (2D); in a continuous pizeo-driven scan with a Pilatus 300k or EigerX $4 \mathrm{M}$ detector, this allows for fast data acquisition and scan times of a few minutes up to half an hour for scan ranges up to $100 \mu \mathrm{m} \times 100 \mu \mathrm{m}$ or more. For such measurements, the Eiger detector is mounted at a distance of about $5.1 \mathrm{~m}$ downstream the sample.

\section{Kirkpatrick-Baez mirror}

While the sample is raster-scanned at a real-space resolution of about $300 \mathrm{~nm}$, in reciprocal space structures of typical length scales down in the range of $2 \mathrm{~nm}-20 \mathrm{~nm}$ are detected. But this diffraction data is not directly inverted numerically to obtain an image of the sample, but rather interpreted quantitatively in $q$-space. Therefore, several algorithms have been developed and tested, e.g. using Fourier transforms for the so-called "streak finder" [6], or by applying principal component analysis to quantify anisotropic scattering in nano-SAXS [7]. Both methods allow one to determine and quantify X-ray scattering from locally ordered structures, e.g. actin fibres in cardiac tissue cells. In contrast to e.g. ptychographic images, the algorithms deliver quantitative parameters to describe the objects of interest in a statistically manner.

With the EigerX detector mounted directly downstream the sample (in a distance closer than $300 \mathrm{~mm}$ ), both WAXS and SAXS signals can be recorded simultaneously, and by correlating Bragg peaks in X-ray Cross Correlation Analysis (XCCA), e.g. the preferential alignment of nanocrystals assembled as a mesocrystal inside polymers can be quantified [8].

\section{Multilayer Zone Plate nano focus}

Thanks to recent advances in optics fabrication, multilayer Laue lenses (MLLs) and multilayer zone plates (MZPs) have emerged, that bring the prospect of few nm beam sizes at hard X-ray energies [9-12]; we are currently exploring the suitability of MZPs with sub-10 nm outermost zone widths for nano-SAXS and nano-WAXS experiments [13].

\section{WAVEGUIDE HOLO-TOMOGRAPHY}

\section{Overview}

Waveguide-improved holography is an imaging technique for $3^{\text {rd }}$ generation synchrotron sources with a limited degree of coherence [14], that can filter the beam to reduce artefacts in image reconstruction [15-16]; as a full-field technique, holography joins naturally with tomography [17-20].

\section{Holography}

$\mathrm{X}$-ray holography is an imaging technique, where the weakly scattering sample imprints a small phase shift onto the wavefield, which is proportional to the local projected electron density (thin sample approximation). Due to interference of the wave-field with itself, even smallest phase distortions yield appreciable intensity variations in the optical near- and far-field. 
From these, the object can be reconstructed e.g. using a direct holographic inversion (very fast and deterministic, but the quality suffers from a twin image), using iterative reconstructions exploiting a priori knowledge (e.g. support constraint; slower and less deterministic), or using the transport of intensity equation or contrast transfer function (measurements in distinct defocus plane are necessary; but very stable and robust). The waveguide enables to record clean holograms with a larger field of view and reduced artefacts compared to Kirkpatrick-Baez mirror systems.

Holography can also be complemented with nano-SAXS measurements, since holography completes missing scattering data at low momentum transfer [5] and it overcomes the mass thickness effect in fluorescence mapping [21].

\section{Tomography}

Using a single rotation axis, waveguide based 2D holography can easily be extended to a tomographic method for 3D (and $4 \mathrm{D}$, if sufficiently high flux allows for time-resolved tomography) [17-20]. We underline the zooming-capability of this scheme, since the field of view / demagnified pixel size depends on the distance between waveguide and sample, and hence can easily be controlled. Typically, the field of view is aligned to values between $10 \mu \mathrm{m}$ and $100 \mu \mathrm{m}$ with about 2000 pixels. Using holo-tomography, biological cells can be imaged in $3 \mathrm{D}$ with a sub-organelle resolution in large tissue [17,21].

\section{CONCLUSIONS \& OUTLOOK}

While a high degree of flexibility in the end-station is a necessary condition to explore and implement new experimental techniques, vibrations are the other side of the coin. Recent progress in optics development promises beam sizes down to the single nano metre, which has to be included in the design of end-stations. Using mechanical simulation software and incorporating experimental data on vibrations (including masses, damping factors, and spring constants), we have found have build a minimal model of the GINIX setup and are now tackling both the sources of vibrations, and are establishing means to reduce their impact.

In the second part, we have summaries recent progress in X-ray imaging at the GINIX setup, in particular using scanning nano-SAXS as a means to quantify local structure and ordering, and waveguide based holography and its extension to holo-tomography; the resolution has been increased to the sub-organelle level of samples in tissue.

The utilisation of X-ray waveguides as coherence filters necessary at $3^{\text {rd }}$ generation sources has shown significant progress, now that a flux of $10^{10}$ photons per second can be achieved; this allows for fast full-field imaging in holography with exposure times of $0.1 \mathrm{~s}$ and below, enabling efficient use of beamtime for tomography. Also the empty beam correction needed for holography is certainly improved, since waveguides show a more Gaussian illumination function as compared to Kirkpatrick-Baez mirror systems. This also holds true for $4^{\text {th }}$ generation sources, that promise two orders of magnitude more coherent flux, and hence illumination times on the few millisecond scale.

\section{REFERENCES \& ACKNOWLEDGEMENTS}

[1] T. Salditt, M. Osterhoff, M. Krenkel, R.N. Wilke, M. Priebe, M. Bartels, S. Kalbfleisch, M. Sprung: Compound focusing mirror and X-ray waveguide optics for coherent imaging and nano-diffraction, Journal of Synchrotron Radiation 22, 867-878 (2015).

[2] S. Kalbfleisch, H. Neubauer, S.P. Krüger, M. Bartels, M. Osterhoff, D.D. Mai, K. Giewekemeyer, B. Hartmann, M. Sprung, T. Salditt: The Göttingen Holography Endstation of Beamline P10 at PETRA III/DESY, AIP Conference Proceedings 1365 (2011).

[3] J. Wallentin, R.N. Wilke, M. Osterhoff, T. Salditt: Simultaneous high-resolution scanning Bragg contrast and ptychographic imaging of a single solar cell nanowire, Journal of Applied Crystallography 48 (2015).

[4] S. Hoffmann-Urlaub, P. Höhne, M. Kanbach, T. Salditt: Advances in fabrication of X-ray waveguides, Microelectronic Engineering 164, 135-138 (2016).

[5] J.D. Nicolas, M. Bernhardt, M. Krenkel, C. Richter, S. Luther, T. Salditt: Combined scanning x-ray diffraction and holographic imaging of cardiomyocytes, J. Appl. Crystallogr. 50, 612-620 (2017).

[6] M. Priebe, M. Bernhardt, C. Blum, M. Tarantola, E. Bodenschatz, T. Salditt: Scanning X-Ray Nanodiffraction on Dictyostelium discoideum, Biophysical Journal 107, 2662-2673 (2014).

[7] M. Bernhardt, J.D. Nicolas, M. Eckermann, B. Eltzner, F. Rehfeldt, T. Salditt: Anisotropic x-ray scattering and orientation fields in cardiac tissue cells, New Journal of Physics, 19, 013012 (2017). 
[8] I.A. Zaluzhnyy, R.P. Kurta, A. André, O.Y. Gorobtsov, M. Rose, P. Skopintsev, I. Besedin, A.V. Zozulya, M. Sprung, F. Schreiber, I.A. Vartanyants, M. Scheele: Quantifying Angular Correlations between the Atomic Lattice and the Superlattice of Nanocrystals Assembled with Directional Linking, Nano Letters 17, 3511-3517 (2017).

[9] A. Kubec, K. Melzer, J. Gluch, S. Niese, S. Braun, J. Patommel, M. Burghammer, A. Leson: Point focusing with flat and wedged crossed multilayer Lause lenses, J. Synchrotron Rad. 24, 413-421 (2017).

[10] S. Bajt, M. Prasciolu, A.J. Morgan, H.N. Chapman, J. Krzywinski, A. Andrejczuk: One dimensional focusing with high numerical aperture multilayer Laue lens, AIP Conference Proceedings 1696, 020049 (2016).

[11] F. Döring, A.L. Robisch, C. Eberl, M. Osterhoff, A. Ruhlandt, T. Liese, F. Schlenkrich, S. Hoffmann, M. Bartels, T. Salditt, H.U Krebs: Sub-5 nm hard X-ray point focusing by a combined Kirkpatrick-Baez mirror and multilayer zone plate, Optics Express 21, 19311-19323 (2013).

[12] H. Yan, Y.S. Chu, E. Nazaretski, J. Kim, H.C. Kang, J.J. Lobardo, W.K.S. Chiu: Quantitative x-ray phase imaging at the nanoscale by multilayer Laue lenses, Scientific Reports 3, 1307 (2013).

[13] M. Osterhoff, J. Soltau, C. Eberl, H.U. Krebs: Faster scanning, higher resolution: New setup for Multilayer Zone Plate Imaging, Proc. SPIE 10389, 29 (this volume, 2017).

[14] M. Osterhoff, T. Salditt: Coherence filtering of x-ray waveguides: analytical and numerical approach, New Journal of Physics 13, 103026 (2011).

[15] J. Hagemann, A.L. Robisch, M. Osterhoff, T. Salditt: Probe reconstruction for holographic X-ray imaging, J. Synchrotron Radiation 24, 498-505 (2017).

[16] C. Homann, T. Hohage, J. Hagemann, A.L. Robisch, T. Salditt: Validity of the empty-beam correction in near-field imaging, Phys. Rev. A 91, 013821 (2015).

[17] C. Dullin, S. dal Monego, E. Larsson, S. Mohammadi, M. Krenkel, C. Garrovo, S. Biffi, A. Lorenzon, A. Markus, J. Napp, T. Salditt, A. Accardo, F. Alves, G. Tromba: Functionalized synchrotron in-line phase-contrast computed tomography: a novel approach for simultaneous quantification of structural alterations and localization of barium-labelled alveolar macrophages within mouse lung samples, Journal of Synchrotron Radiation 22, 143-155 (2015).

[18] M. Bartels, M. Krenkel, J. Haber, R. N. Wilke, T. Salditt: X-Ray Holographic Imaging of Hydrated Biological Cells in Solution, Phys. Rev. Lett. 114, 048103 (2015).

[19] R.N. Wilke, M. Hoppert, M. Krenkel, M. Bartels, T. Salditt: Quantitative X-ray phase contrast waveguide imaging of bacterial endospores, J. Appl. Cryst. 48, 464-476 (2015).

[20] M. Krenkel, A. Markus, M. Bartels, C. Dullin, F. Alves, T. Salditt: Phase-contrast zoom tomography reveals precise locations of macrophages in mouse lungs, Scientific Reports 5, 09973 (2015).

[21] A.D. Surowka, M. Töpperwien, M. Bernhardt, J.D. Nicolas, M. Osterhoff, T. Salditt, D. Adamek, M. Szczerbowska-Boruchowska: Combined in-situ imaging of structural organization and elemental composition of substantia nigra neurons in the elderly, Talanta 161,368-376 (2016).

\section{Acknowledgments}

We thank Bastian Hartmann, Eiryn Schroeder and Daniel Weschke for excellent engineering support, and DESY Photon Science for commissioning beamtime. We acknowledge funding by Bundesministerium für Bildung und Forschung through Verbundforschung projects 05K13MG4,05K16MGA and 05K16MGB and Deutsche Forschungsgemeinschaft through SFB 755 "Nanoscale Photonic Imaging". 